\title{
Accurate Assignments of Excited-State Resonance Raman Spectra: A Benchmark Study Combining Experiment and Theory
}

\author{
Matthew S. Barclay, ${ }^{\dagger}$ Timothy J. Quincy, ${ }^{\dagger}$ David B. Williams-Young, ${ }^{\ddagger}$ Marco Caricato, ${ }^{*} \dagger \odot$ \\ and Christopher G. Elles*, ${ }^{\dagger}$ \\ ${ }^{\dagger}$ Department of Chemistry, University of Kansas, Lawrence, Kansas 66045, United States \\ ${ }^{\ddagger}$ Department of Chemistry, University of Washington, Seattle, Washington 98195, United States
}

\section{Supporting Information}

ABSTRACT: Femtosecond stimulated Raman scattering (FSRS) probes the structural dynamics of molecules in electronically excited states by following the evolution of the vibrational spectrum. Interpreting the dynamics requires accurate assignments to connect the vibrational bands with specific nuclear motions of an excited molecule. However, the assignment of FSRS signals is often complicated by mode-specific resonance enhancement effects that are difficult to calculate for molecules in electronically excited states. We present benchmark results for a series of eight arylsubstituted thiophene derivatives to show that calculated off-resonance Raman spectra

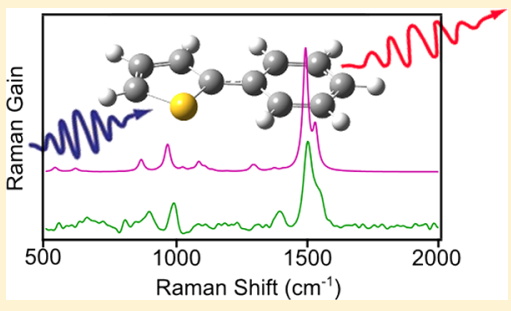
can be used to assign experimental bands on the basis of a comparison of structurally similar compounds and careful consideration of the resonance condition. Importantly, we show that direct comparison with the off-resonant calculations can lead to incorrect assignments of the experimental spectrum if the resonance condition is neglected. These results highlight the importance of resonance enhancement effects in assigning FSRS spectra.

\section{INTRODUCTION}

Femtosecond stimulated Raman scattering (FSRS) is a sensitive method for probing excited-state dynamics. ${ }^{1,2}$ The technique reveals the structural evolution of a molecule by monitoring changes in the vibrational spectrum as a function of time following optical excitation. Correctly interpreting the excitedstate dynamics therefore requires accurate assignments of the bands in the transient Raman spectrum. While some assignments are possible based on simple chemical intuition or comparison with known ground-state spectra, many assignments require at least some level of comparison with excitedstate calculations. In general, the excited-state vibrational frequencies are relatively easy to calculate, but the Raman intensities are more difficult to obtain due to the need to calculate changes of the excited-state polarizability tensor along each of the normal-mode coordinates. The intensities are important for comparison with experiment, because the frequencies alone may not provide enough information in regions of the spectrum with a relatively high density of states. An important and often overlooked complication is that experimental FSRS measurements often rely on mode-specific resonance enhancement effects in order to compensate for the small population of molecules in the excited state, ${ }^{1-3}$ or to selectively probe a single species in the transient spectrum. ${ }^{4}$ Resonance enhancement effects are difficult to include in excited-state Raman calculations, because of challenges in treating higher-lying electronic states that are responsible for the resonant condition.

This paper examines the assignment of excited-state Raman bands by comparing off-resonance calculations with experimental resonance-enhanced FSRS spectra for a series of $\pi$ - conjugated molecules. The resonance enhancements are vibrational-mode-dependent, leading to relative intensities in the experimental spectra that can be very different from the offresonance calculations. ${ }^{5-7}$ We show that a comparison across the entire series of benchmark compounds facilitates the assignment of the experimental transitions, even though a direct comparison of the calculated off-resonance intensities with the experimental spectrum for only a single molecule can be misleading and could result in incorrect assignments. The vibrational frequencies depend on the structure of the molecule, but modes with similar nuclear motions are enhanced for all of the molecules in the series because resonant excitation into the excited-state absorption bands causes a similar change of the $\pi$-conjugated structure for each molecule.

Our calculations neglect the resonance condition in an attempt to find a balance between accuracy and computational efficiency. More sophisticated methods that explicitly treat the resonance enhancement by directly simulating the third-order polarization response of an excited molecule are computationally expensive and would be prohibitive for calculating the spectra for a series of relatively large compounds. ${ }^{8-15}$ Instead, our off-resonance calculations take advantage of efficient analytical second derivatives of the energy that are now available for time-dependent density functional theory (TDDFT) in order to calculate the excited-state polarizability. ${ }^{16-19}$ Specifically, we calculate the excited-state Raman activities using single numerical differentiation of the (analytical) static polarizability tensor along each of the excited-

Received: September 24, 2017

Published: September 27, 2017 
state normal-mode coordinates. ${ }^{20}$ This method gives excitedstate Raman spectra with DFT accuracy at relatively low computational cost. Earlier attempts to calculate Raman spectra from the excited-state polarizability required numerical differentiation of the electric dipole moment with respect to the electric field, in addition to the normal-mode displacements. $^{21-23}$

The series of compounds in our benchmark study consists of aryl-substituted thiophenes that are representative $\pi$-conjugated systems. Thiophene derivatives have been the subject of previous transient absorption ${ }^{24-26}$ and FSRS studies ${ }^{27-30}$ because of their importance as building blocks for photoactive polymers and molecular switches. Conjugated thiophenes also undergo rapid intersystem crossing (ISC), which is an important property for charge separation and transport in conductive organic materials. ${ }^{31-34}$ The ISC rates depend on the structure of the compound and range from tens to hundreds of picoseconds. ${ }^{26}$ We compare the calculated and experimental Raman spectra for both singlet and triplet excited states of each molecule in order to evaluate resonance enhancement effects in the different spin states. In general, we find that the calculated off-resonance intensities are in better agreement with resonance-enhanced experimental spectra for triplet states compared with singlet states. Comparing trends in the calculated and experimental spectra across the entire series of molecules allows us to make accurate assignments for both the singlet and triplet states using relatively low-cost off-resonance excited-state calculations, provided the electronic resonance condition is taken into consideration.

\section{METHODS}

The series of aryl-substituted thiophene derivatives includes 2,5-diphenylthiophene (DPT), 2-phenylthiophene (PT), 2methyl-5-phenylthiophene (MPT), 2,4-dimethyl-5-phenylthiophene (DMPT), 2,5-terthiophene (TT), 2,2'-bithiophene (2,2'-BT), 2,3'-bithiophene (2,3'-BT), and 3,4'-dihexyl-2,2'bithiophene (DHBT). All compounds were obtained commercially and used as received by dissolving in cyclohexane (1-10 $\mathrm{mM})$.

Transient absorption and stimulated Raman measurements use the modified output of an amplified Ti:sapphire laser (Coherent Legend Elite). An optical parametric amplifier (OPA) and two stages of nonlinear frequency conversion provide $\sim 80 \mathrm{fs}$ actinic pulses at $310 \mathrm{~nm}$ that are attenuated to $0.5-0.9 \mu \mathrm{J}$ at the sample. The frequency-doubled output of a second OPA passes through a $25 \mathrm{~mm}$ long BBO crystal to produce ps-duration Raman pump pulses via second harmonic generation (SHG), followed by a $4 \mathrm{f}$ spectral filter for additional spectral narrowing and rectification. ${ }^{35,36}$ The resulting Raman pump pulses are tunable over the range 350-650 nm, with bandwidths of $\sim 15-30 \mathrm{~cm}^{-1}$ and pulse energies up to $1 \mu \mathrm{J}$. The fs broadband probe pulses come from continuum generation in a circularly translating $\mathrm{CaF}_{2}$ substrate. After the sample, a spectrograph disperses the probe light onto a linear CCD array detector with 2048 pixels. We use an adapted version of the scanning multichannel technique developed by Challa et al. ${ }^{37}$ in order to reduce systematic noise from the variable pixel response of the CCD array. Specifically, we obtain FSRS spectra by averaging measurements at five different grating angles in the spectrograph with approximately $20 \mathrm{~cm}^{-1}$ shift between individual spectra. The baseline subtraction method is described in the Supporting Information (SI). We record off-resonant ground-state Raman spectra using a commercial Raman spectrometer (StellarNet) with $785 \mathrm{~nm}$ laser source and $4 \mathrm{~cm}^{-1}$ resolution. We remove solvent bands from the ground- and excited-state Raman spectra by subtraction. The experimental procedures are described in more detail elsewhere. ${ }^{38}$

We calculate off-resonance Raman spectra by applying the polarizability derivative approach of Neugebauer et al. ${ }^{20}$ In the case of the excited-state Raman calculations, we first obtain the $\mathrm{S}_{1}$ or $\mathrm{T}_{1}$ minimum energy geometry and normal-mode coordinates analytically using TDDFT with the B3LYP functional ${ }^{39-41}$ and aug-cc-pVDZ basis set. ${ }^{42}$ The Raman activities are then obtained by taking numerical derivatives of the excited-state static polarizability tensor with respect to displacements along the normal-mode coordinates. We determine the optimum step size for the normal-mode displacements based on comparison with analytical groundstate Raman calculations for several small molecules and various levels of theory (Figure S1 in the SI). Numerical differentiation at the standardized step size gives Raman activities that are nearly identical to the analytical results for $S_{0}$. We use the same step size for the excited-state calculations. A recent benchmarking study by Staniszewska et al. ${ }^{43}$ motivates our choice of B3LYP/aug-cc-pVDZ. All calculations were performed using a development version of the GAUSSIAN suite of programs. 4

\section{RESULTS AND DISCUSSION}

Transient Absorption Spectroscopy and Excited-State Dynamics. Figure 1 shows the transient electronic absorption spectra for the series of aryl-substituted thiophene derivatives following $\pi \rightarrow \pi^{*}$ excitation with an actinic pulse near $310 \mathrm{~nm}$. The transient spectrum of each compound is characterized by strong excited-state absorption (ESA) and stimulated emission (SE) bands of the singlet $S_{1}$ excited state at short time delays. Although not shown here, the spectra evolve on a time scale of a few ps due to structural relaxation and vibrational cooling, including planarization of the molecule in response to the increased double-bond character of the inter-ring $\mathrm{C}-\mathrm{C}$ bonds in the $\pi \pi^{*}$ state. $^{26}$ All of the molecules eventually undergo intersystem crossing (ISC) to a triplet excited state, $\mathrm{T}_{1}$, at longer delay times. The intersystem crossing times $\left(\tau_{\text {ISC }}\right)$ vary from 22 to 267 ps, depending on the structure of the molecule, and are summarized in Table 1 . The triplet states are relatively long-lived (>1 ns) and have ESA bands at shorter wavelengths compared with the singlet excited states.

The relatively fast $S_{1} \rightarrow T_{1}$ relaxation times are a result of strong spin-orbit coupling of the thiophene sulfur atom, with nonplanar geometries accelerating the ISC. ${ }^{26}$ Accordingly, the ISC rate increases for molecules with more than one sulfur atom, and for molecules that have steric interactions that destabilize the planar geometry of the $S_{1}$ excited state. The three-ring compounds DPT and TT have the longest $\tau_{\text {ISC }}$ due to increasing conjugation that favors a more planar structure in the excited state. ${ }^{45}$

In order to measure the stimulated Raman spectra of the singlet and triplet excited states, we tune the Raman pump wavelength close to the maximum of the respective ESA band (red and green arrows in Figure 1), and we adjust the delay between actinic pump and Raman probe pulses accordingly. The $S_{1}$ spectra are measured at delays ranging from 5 to 30 ps to allow vibrational relaxation and cooling in the singlet state, but before ISC begins to populate the triplet state. The $T_{1}$ spectra are obtained at delays that are about 4 times longer than 

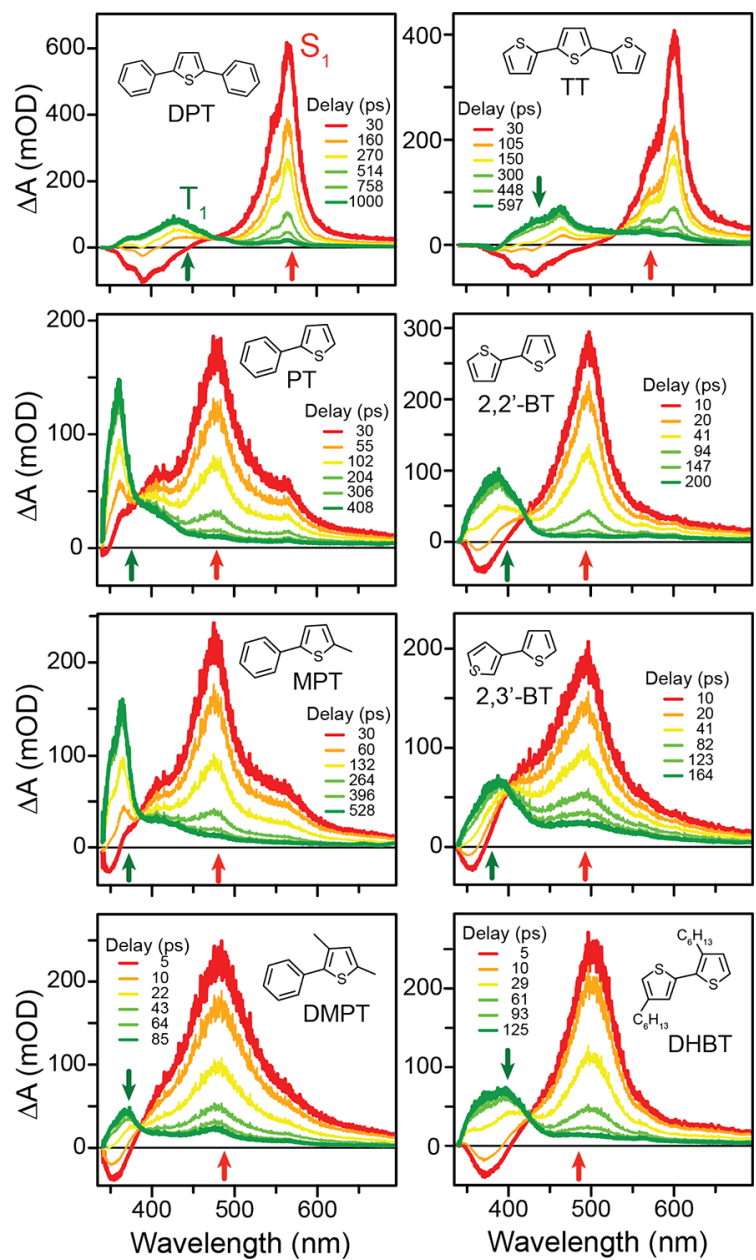

Figure 1. Transient absorption spectra showing the evolution from $S_{1}$ (red) to $\mathrm{T}_{1}$ (green) excited states for the series of aryl-substituted thiophene derivatives. Red and green arrows indicate the Raman pump wavelengths for stimulated resonance Raman measurements of the singlet and triplet excited states, respectively.

Table 1. Intersystem Crossing Lifetimes and FSRS Delay Times $^{a}$

\begin{tabular}{cccc} 
& $\tau_{\text {ISC }}$ & $\mathrm{S}_{1}$ delay & $\mathrm{T}_{1}$ delay \\
DPT & 267 & 30 & 1000 \\
PT & 102 & 30 & 410 \\
MPT & 132 & 30 & 530 \\
DMPT & 22 & 5 & 85 \\
TT & 149 & 50 & 595 \\
$2,2^{\prime}-\mathrm{BT}$ & 41 & 10 & 200 \\
$2,3^{\prime}-\mathrm{BT}$ & 37 & 10 & 165 \\
DHBT & 29 & 5 & 125 \\
${ }^{a}$ All times in ps. & & & \\
\hline
\end{tabular}

the ISC time of each molecule to ensure significant conversion to the triplet state. Table 1 gives the experimental time delays for the singlet and triplet FSRS measurements.

Ground-State Raman Spectroscopy. Before examining the excited-state Raman spectra, we compare the calculated and experimental spectra for the ground electronic state of each molecule in Figure 2. The $S_{0}$ spectra are off-resonant for both experiment and theory. Comparing the calculated ground-state spectra with experiment provides an important reference point for estimating the accuracy of the DFT calculations. The

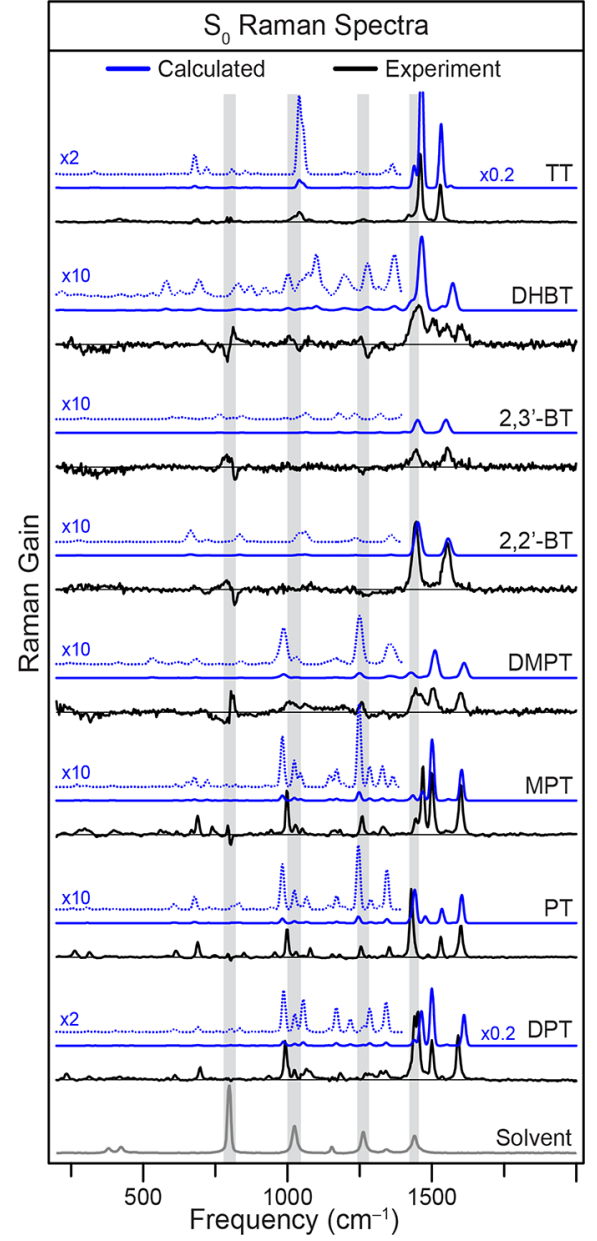

Figure 2. Raman spectra of the ground electronic states. Calculated spectra (blue lines) are shown on a common intensity scale, except TT and DPT are reduced by a factor of 5 . The the low-frequency region of each spectrum is also shown on an expanded scale (dotted blue lines, $10 \times)$. Experimental spectra in cyclohexane solution (black lines) are measured off-resonance using either spontaneous Raman at $785 \mathrm{~nm}$ (TT, MPT, PT, DPT) or stimulated Raman at $370 \mathrm{~nm}$ (DHBT, 2,3'BT, 2,2'-BT, DMPT). The solvent spectrum is shown for reference and to indicate where there may be subtraction errors in the experimental spectra.

accuracy in the ground state is the best that can be expected for the excited-state calculations based on inherent limitations of the method and basis set (B3LYP/aug-cc-pVDZ). To facilitate the comparison between ground and excited states, the groundstate calculations use the same numerical differentiation along the normal-mode coordinates that we use for the excited states below, even though analytical derivatives are available for the ground state. The numerical derivatives give Raman activities that differ from the analytical derivative results by $<0.05 \%$.

As expected, the calculated vibrational frequencies overestimate the experimental values; therefore, we apply an empirical frequency scaling factor. A scaling factor of 0.976 gives the best agreement across the entire series of molecules (see below), consistent with the commonly accepted value of 0.970 for this level of theory and basis set. ${ }^{46}$ We also broaden each transition in the calculated spectrum with a $15 \mathrm{~cm}^{-1}$ Gaussian in order to simulate the experimental line widths in Figure 2. 
The ground-state calculations generally are in good agreement with the experimental Raman spectra for $S_{0}$. In most cases, the calculations reproduce the experimental frequencies within $\sim 20 \mathrm{~cm}^{-1}$ and the intensities within an order of magnitude, which is typical for DFT. ${ }^{4-49}$ For example, the calculations accurately reproduce the dominant $\mathrm{C}=\mathrm{C}$ ethylenic stretches in the frequency range $\sim 1400-1700 \mathrm{~cm}^{-1}$. The only discrepancies in this region are for DHBT, DMPT, and MPT, each of which has one or two bands with slightly higher relative intensity in the experiments than predicted by theory. We note that these three are the only alkyl substituted compounds and that the orientations of the methyl and hexyl side groups might affect the calculated spectra. Although the conformational dependence is important, it is beyond the scope of this work.

The $S_{0}$ calculations also slightly underestimate the relative intensities of some of the lower-frequency modes. Figure 2 shows the low-frequency region of the calculated spectra on an expanded scale in order to facilitate the comparison in this region. Although the relative intensities are underestimated by up to an order of magnitude, the calculated frequencies are in very good agreement with experiment. The most notable discrepancy is the relatively strong experimental band just below $1000 \mathrm{~cm}^{-1}$ for the phenyl-substituted thiophenes MPT, PT, and DPT, where the experimental intensities more closely match the spectra on the $10 \times$ expanded scale. Previously reported computational and experimental spectra for the $S_{0}$ state of DPT also have an approximately 10 -fold difference in the relative intensity of the $\sim 1000 \mathrm{~cm}^{-1}$ band, consistent with our observation. ${ }^{49}$

Resonance Raman Spectroscopy of $T_{1}$. We examine the calculated and experimental Raman spectra of the triplet excited states in Figure 3. As is the case for the $S_{0}$ spectra, the $T_{1}$ calculations use the numerical differentiation procedure, even though $\mathrm{T}_{1}$ is the lowest triplet state and therefore can be treated as a ground state to obtain analytical derivatives of the polarizability. All of the spectra from the off-resonant $T_{1}$ calculations are dominated by a single, strong band near 1500-1550 $\mathrm{cm}^{-1}$, with much weaker transitions at lower frequencies. We obtain the best agreement with the experimental frequencies by using a scaling factor of 0.964 , as discussed in more detail below. We apply $25 \mathrm{~cm}^{-1}$ Gaussian broadening to the calculated transitions in the figure in order to match the experimental resolution.

Unlike the $S_{0}$ spectra, the experimental $T_{1}$ spectra are obtained with Raman pump wavelengths that are resonant with an excited-state (triplet-triplet) absorption band. Despite the resonance condition, the calculated spectra are generally in good agreement with experiment, including the most prominent transitions in the ethylenic stretching region, 1400-1700 $\mathrm{cm}^{-1}$. The bithiophene compounds (DHBT and BT) and terthiophene (TT) have only a single, strong band in this region, whereas the spectra of all four phenyl-substituted thiophenes have an additional shoulder. The shoulders appear as distinct transitions in the calculated spectra of MPT and PT.

Compared with the ethylenic stretching modes, many of the weaker transitions in the lower-frequency regions of the experimental $T_{1}$ spectra are more difficult to distinguish from noise. However, there are several transitions that are reproduced by the calculations, such as the pair of peaks representing the phenyl stretching modes near 894 and 986 $\mathrm{cm}^{-1}$ for DMPT, MPT, and PT. In a few cases, the experimental bands are much stronger than the calculations predict. For example, the prominent bands near $343 \mathrm{~cm}^{-1}$ in

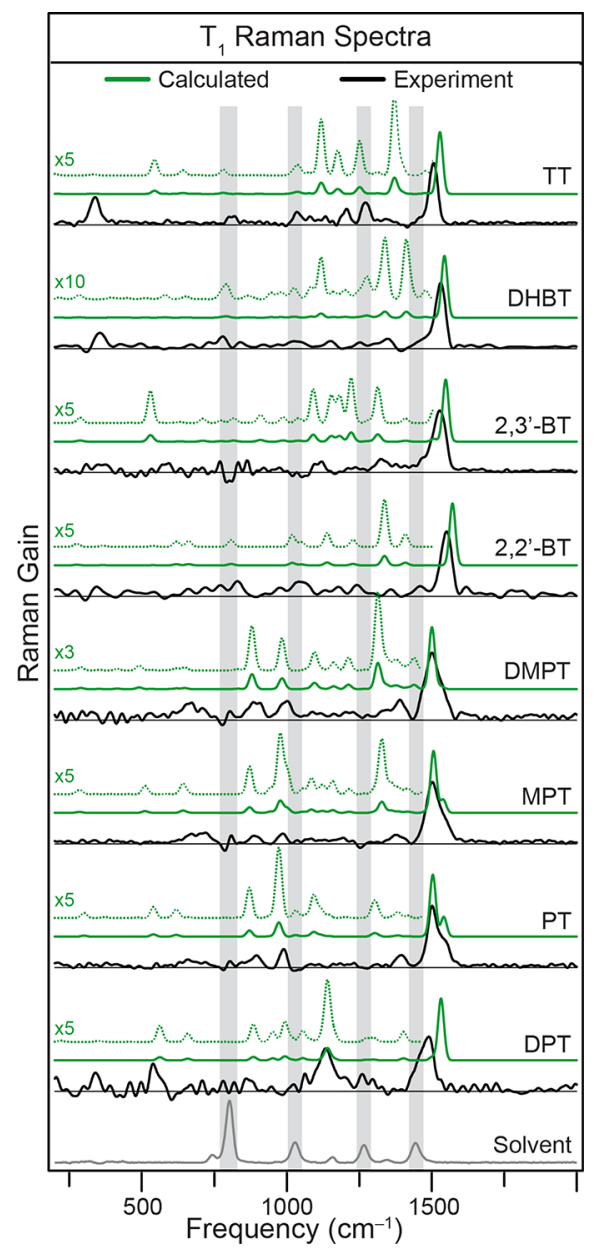

Figure 3. Raman spectra of the triplet states. Calculated spectra (green lines) are normalized to the most intense peak of each molecule, with the low-frequency region of each spectrum also shown on an expanded scale (dotted green lines). Experimental spectra (black) are measured in resonance with the $T_{1}-T_{n}$ absorption band of each compound, and are also normalized to the most intense peak of each spectrum. The solvent spectrum is shown for reference and to indicate where there may be subtraction errors in the experimental spectra.

the experimental spectra of TT and DHBT are significantly stronger than any of the calculated modes in that region.

The significantly higher experimental intensities of the lowfrequency bands is probably due to the resonance condition. Analogous with ground-state resonance Raman spectroscopy, ${ }^{6,7}$ we expect an additional enhancement for vibrational modes that have a large displacement in the upper triplet states relative to the minimum-energy structure of $\mathrm{T}_{1} \cdot{ }^{32}$ Resonance enhancement also explains the emergence of the broad Raman features near $650-750 \mathrm{~cm}^{-1}$ in the experimental spectra of DMPT, $\mathrm{MPT}$, and PT, as well as the relative increase in the intensity of the experimental bands of DPT near 340, 541, and $1134 \mathrm{~cm}^{-1}$. Overall, the generally good agreement between the off-resonant calculations and the resonant experimental stimulated Raman spectra suggest that the resonance condition does not significantly affect the triplet spectra, with the exception of a few specific low-frequency modes.

Resonance Raman Spectroscopy of $S_{1}$. The Raman spectra of the singlet excited states are the main focus of this work. Figure 4 shows the experimental and calculated spectra for the $S_{1}$ state of each molecule. The calculated spectra include 


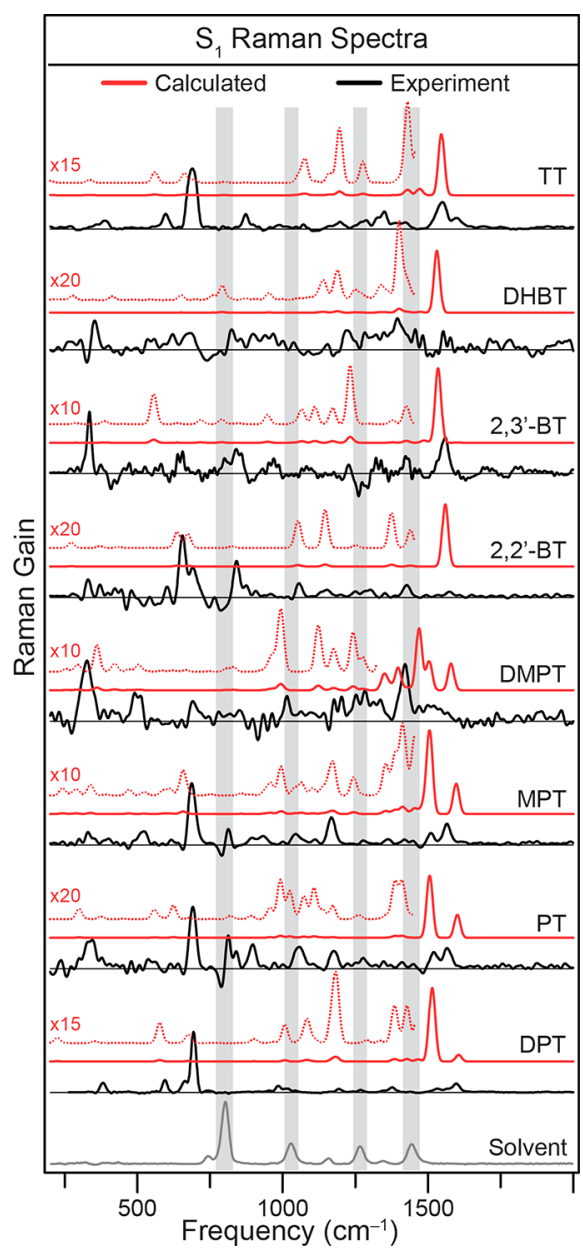

Figure 4. Raman spectra of the $S_{1}$ excited states. Calculated spectra (red lines) are normalized to the most intense peak of each molecule, with the low-frequency region of each spectrum also shown on an expanded scale (dotted red lines). Experimental spectra (black lines) are measured in resonance with the strong $S_{1}-S_{n}$ absorption band of each compound, and they are also normalized to the most intense peak of each spectrum. The solvent spectrum is shown for reference and to indicate where there may be subtraction errors in the experimental spectra.

$25 \mathrm{~cm}^{-1}$ Gaussian broadening and a frequency scaling factor of 0.977. As before, all of the strongest bands in the calculated offresonance spectra are in the ethylenic range, $1400-1700 \mathrm{~cm}^{-1}$, with the lower-frequency transitions having much lower intensity. In sharp contrast with the $S_{0}$ and $T_{1}$ results, however, some of the low-frequency modes are among the strongest bands in the experimental spectra of the $S_{1}$ excited states.

The stark discrepancy between the relative intensities in the experimental and calculated spectra indicates that the resonance condition plays a significant role for the $S_{1}$ excited states. Based on the time-dependent formalism for resonance Raman spectroscopy, ${ }^{6,7}$ the resonance enhancement of a given vibrational mode depends on the relative displacement of the higher-lying excited-state potential energy surface along the normal-mode coordinate of the lower-lying electronic state. In the case of the singlet excited-state FSRS spectra, the resonance enhancement comes from the displacement of the molecule in the $S_{n}$ excited states relative to the initial $S_{1}$ state. We examine this resonance condition in more detail elsewhere, ${ }^{38}$ but we note that all of the compounds in this study have similar ESA bands due to secondary $\pi$ excitation from $S_{1}$. Therefore, we predict the strongest enhancements for vibrational modes that are most sensitive to changes in the occupancy of conjugated $\pi$ and $\pi^{*}$ orbitals.

Some of the most notable resonance enhancements in the experimental $S_{1}$ Raman spectra include the bands in the range $650-700 \mathrm{~cm}^{-1}$ for TT, 2,2'-BT, MPT, PT, and DPT, and near $333 \mathrm{~cm}^{-1}$ for DHBT, 2,3'-BT, and DMPT. These are the strongest bands in each of the experimental spectra, even though the off-resonant calculations show only very weak transitions below $\sim 1400 \mathrm{~cm}^{-1}$. Despite neglecting the resonance condition in the calculations, we can still use the calculated spectra to help assign the experimental transitions on the basis of the frequencies. However, we show below that it is also important to consider the specific nuclear motions associated with each vibration in order to determine which transitions gain intensity from the resonance condition. Figure 4 shows each of the calculated spectra on an expanded scale below $\sim 1450 \mathrm{~cm}^{-1}$ to facilitate the comparison.

Resonance Raman Band Assignments for $S_{1}$. As a first step in assigning the $S_{1}$ transitions, we evaluate the accuracy of the calculated frequencies by considering the empirical scaling factors in more detail. The scaling factors reported above for the $S_{0}, T_{1}$, and $S_{1}$ states were obtained by comparing the most prominent experimentally observed transitions with the calculated frequencies for all of the molecules. Rather than making the assignments and frequency comparisons by inspection alone, we compare the calculated vibrational motions across the series of molecules in order to refine our initial assignments, as described in the example below. Table 2 lists the assigned transitions. The resulting calibration curves in Figure 5 include a total of 30-40 transitions for each state and give scaling factors for all three states $\left(0.976 \pm 0.001\right.$ for $S_{0}$, $0.964 \pm 0.003$ for $T_{1}$, and $0.977 \pm 0.003$ for $S_{1}$ ) that are in close agreement with the commonly accepted value of 0.970 for ground-state calculations using B3LYP/aug-cc-pVDZ. ${ }^{46}$

The strong correlation between experimental and theoretical frequencies suggests that, on average, the calculations accurately reproduce the excited-state vibrations within $\sim 20-30 \mathrm{~cm}^{-1}$ after applying the empirical scaling factor. Thus, relatively isolated vibrations are easy to assign on the basis of frequencies alone. More congested regions of the spectrum require closer inspection and careful consideration of resonance enhancement effects. For example, Figure 6 shows the $450-900 \mathrm{~cm}^{-1}$ region of the $S_{1}$ spectrum for MPT, PT, and DPT. In contrast with the single, strong experimental bands near $690 \mathrm{~cm}^{-1}$, the strongest calculated transitions of the three molecules vary by almost 100 $\mathrm{cm}^{-1}$. Although it is tempting to assign the experimental transitions on the basis of the most prominent bands in the calculated spectra, the variation of the calculated frequencies compared with the relatively invariant experimental values suggests that mode-specific resonance enhancement effects are important. In order to make the assignments, we exploit the fact that only transitions to the totally symmetric vibrational modes of a molecule are enhanced by resonant excitation. ${ }^{49,50}$ Transitions to nontotally symmetric modes will not contribute to strongly resonance-enhanced FSRS spectra like those considered here. Furthermore, resonance enhancement effects are most pronounced for vibrational modes that are FranckCondon active in the resonant (upper) electronic state.

The $S_{1}$ state of DPT has 15 calculated vibrations in the range $500-800 \mathrm{~cm}^{-1}$. Twelve of those vibrations are nontotally symmetric modes that can be neglected using symmetry 
Table 2. Vibrational Band Assignments ${ }^{a}$

\begin{tabular}{|c|c|c|c|c|c|c|c|c|c|}
\hline \multirow[b]{2}{*}{ molecule } & \multicolumn{3}{|c|}{$\mathrm{S}_{0}$} & \multicolumn{3}{|c|}{$\mathrm{T}_{1}$} & \multicolumn{3}{|c|}{$\mathrm{S}_{1}$} \\
\hline & mode & calcd & exptl & mode & calcd & exptl & mode & calcd & exptl \\
\hline \multirow[t]{7}{*}{ TT } & $\nu_{54}$ & 1532 & 1528 & $\nu_{55}$ & 1528 & 1506 & $\nu_{55}$ & 1546 & 1547 \\
\hline & $\nu_{52}$ & 1464 & 1459 & $\nu_{47}$ & 1251 & 1272 & $\nu_{52}$ & 1430 & 1417 \\
\hline & $\nu_{50}$ & 1439 & 1417 & $\nu_{45}$ & 1176 & 1207 & $\nu_{45}$ & 1196 & 1197 \\
\hline & $\nu_{25}$ & 720 & 741 & $\nu_{38}$ & 1037 & 1034 & $\nu_{32}$ & 847 & 873 \\
\hline & $\nu_{23}$ & 679 & 678 & $\nu_{30}$ & 781 & 810 & $\nu_{25}$ & 695 & 693 \\
\hline & & & & $\nu_{6}$ & 331 & 339 & $\nu_{17}$ & 560 & 597 \\
\hline & & & & & & & $\nu_{10}$ & 336 & 388 \\
\hline \multirow[t]{4}{*}{ DHBT } & $\nu_{118}$ & 1465 & 1454 & $\nu_{120}$ & 1544 & 1531 & $\nu_{120}$ & 1531 & 1562 \\
\hline & & & & $\nu_{98}$ & 1337 & 1348 & $\nu_{103}$ & 1400 & 1394 \\
\hline & & & & $\nu_{51}$ & 787 & 780 & $\nu_{41}$ & 652 & 685 \\
\hline & & & & $\nu_{26}$ & 287 & 355 & $\nu_{31}$ & 414 & 354 \\
\hline \multirow[t]{6}{*}{$2,3^{\prime}-\mathrm{BT}$} & $\nu_{36}$ & 1549 & 1554 & $\nu_{36}$ & 1548 & 1528 & $\nu_{36}$ & 1535 & 1560 \\
\hline & $\nu_{34}$ & 1451 & 1446 & $\nu_{33}$ & 1314 & 1326 & $\nu_{34}$ & 1426 & 1422 \\
\hline & & & & $\nu_{28}$ & 1091 & 1108 & $\nu_{28}$ & 1111 & 1082 \\
\hline & & & & $\nu_{7}$ & 384 & 349 & $\nu_{20}$ & 826 & 839 \\
\hline & & & & & & & $\nu_{15}$ & 636 & 645 \\
\hline & & & & & & & $\nu_{7}$ & 387 & 334 \\
\hline \multirow[t]{7}{*}{$2,2^{\prime}-\mathrm{BT}$} & $\nu_{36}$ & 1557 & 1550 & $\nu_{36}$ & 1572 & 1551 & $\nu_{36}$ & 1560 & 1575 \\
\hline & $\nu_{34}$ & 1451 & 1446 & $\nu_{27}$ & 1050 & 1043 & $\nu_{35}$ & 1439 & 1427 \\
\hline & & & & $\nu_{7}$ & 365 & 335 & $\nu_{29}$ & 1146 & 1152 \\
\hline & & & & & & & $\nu_{26}$ & 1056 & 1056 \\
\hline & & & & & & & $\nu_{20}$ & 826 & 841 \\
\hline & & & & & & & $\nu_{16}$ & 674 & 690 \\
\hline & & & & & & & $\nu_{14}$ & 637 & 656 \\
\hline \multirow[t]{6}{*}{ DMPT } & $\nu_{57}$ & 1611 & 1601 & $\nu_{56}$ & 1501 & 1502 & $\nu_{54}$ & 1469 & 1420 \\
\hline & $\nu_{54}$ & 1511 & 1507 & $\nu_{50}$ & 1386 & 1392 & $\nu_{43}$ & 1276 & 1282 \\
\hline & $\nu_{49}$ & 1427 & 1448 & $\nu_{34}$ & 983 & 999 & $\nu_{22}$ & 667 & 690 \\
\hline & $\nu_{42}$ & 1249 & 1255 & $\nu_{28}$ & 881 & 894 & $\nu_{16}$ & 504 & 496 \\
\hline & $\nu_{36}$ & 1030 & 1059 & & & & $\nu_{14}$ & 422 & 326 \\
\hline & $\nu_{32}$ & 987 & 1007 & & & & & & \\
\hline \multirow[t]{9}{*}{$\mathrm{MPT}$} & $\nu_{50}$ & 1603 & 1599 & $\nu_{49}$ & 1506 & 1501 & $\nu_{50}$ & 1597 & 1564 \\
\hline & $\nu_{47}$ & 1501 & 1499 & $\nu_{44}$ & 1384 & 1379 & $\nu_{49}$ & 1506 & 1511 \\
\hline & $\nu_{46}$ & 1468 & 1470 & $\nu_{31}$ & 978 & 986 & $\nu_{42}$ & 1368 & 1363 \\
\hline & $\nu_{38}$ & 1248 & 1260 & $\nu_{24}$ & 872 & 889 & $\nu_{36}$ & 1160 & 1167 \\
\hline & $\nu_{29}$ & 982 & 997 & & & & $\nu_{32}$ & 1041 & 1044 \\
\hline & $\nu_{17}$ & 677 & 689 & & & & $\nu_{20}$ & 670 & 688 \\
\hline & $\nu_{16}$ & 654 & 667 & & & & $\nu_{13}$ & 524 & 522 \\
\hline & $\nu_{15}$ & 613 & 616 & & & & $\nu_{10}$ & 391 & 330 \\
\hline & $\nu_{9}$ & 389 & 399 & & & & & & \\
\hline \multirow[t]{11}{*}{$\mathrm{PT}$} & $\nu_{43}$ & 1604 & 1599 & $\nu_{42}$ & 1504 & 1501 & $\nu_{43}$ & 1601 & 1566 \\
\hline & $\nu_{41}$ & 1535 & 1530 & $\nu_{39}$ & 1384 & 1394 & $\nu_{42}$ & 1506 & 1521 \\
\hline & $\nu_{39}$ & 1441 & 1429 & $\nu_{28}$ & 975 & 989 & $\nu_{35}$ & 1265 & 1277 \\
\hline & $\nu_{37}$ & 1344 & 1351 & $\nu_{22}$ & 871 & 895 & $\nu_{32}$ & 1138 & 1176 \\
\hline & $\nu_{34}$ & 1245 & 1253 & & & & $\nu_{29}$ & 1056 & 1056 \\
\hline & $\nu_{26}$ & 982 & 997 & & & & $\nu_{23}$ & 891 & 897 \\
\hline & $\nu_{14}$ & 678 & 689 & & & & $\nu_{17}$ & 679 & 690 \\
\hline & $\nu_{11}$ & 604 & 612 & & & & $\nu_{11}$ & 558 & 537 \\
\hline & $\nu_{10}$ & 583 & 583 & & & & $\nu_{7}$ & 374 & 343 \\
\hline & $\nu_{5}$ & 307 & 315 & & & & & & \\
\hline & $\nu_{4}$ & 254 & 264 & & & & & & \\
\hline \multirow[t]{9}{*}{$\mathrm{DPT}$} & $\nu_{68}$ & 1611 & 1591 & $\nu_{68}$ & 1532 & 1490 & $\nu_{69}$ & 1606 & 1598 \\
\hline & $\nu_{64}$ & 1500 & 1500 & $\nu_{52}$ & 1141 & 1134 & $\nu_{67}$ & 1519 & 1532 \\
\hline & $\nu_{62}$ & 1464 & 1446 & $\nu_{18}$ & 562 & 539 & $\nu_{59}$ & 1384 & 1377 \\
\hline & $\nu_{45}$ & 1056 & 1067 & $\nu_{10}$ & 350 & 340 & $\nu_{53}$ & 1186 & 1193 \\
\hline & $\nu_{41}$ & 988 & 991 & & & & $\nu_{40}$ & 969 & 984 \\
\hline & $\nu_{23}$ & 687 & 698 & & & & $\nu_{25}$ & 675 & 693 \\
\hline & $\nu_{19}$ & 609 & 610 & & & & $\nu_{18}$ & 577 & 594 \\
\hline & $\nu_{18}$ & 589 & 585 & & & & $\nu_{10}$ & 353 & 382 \\
\hline & $\nu_{10}$ & 335 & 351 & & & & & & \\
\hline
\end{tabular}


Table 2. continued

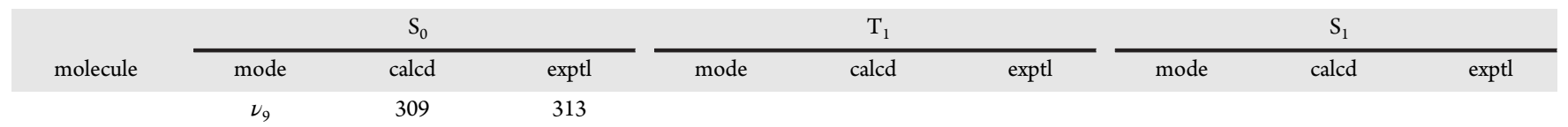

${ }^{a}$ Calculated frequencies have been multiplied by a scaling factor of 0.976 for $S_{0}, 0.964$ for $T_{1}$, and 0.977 for $S_{1}$.

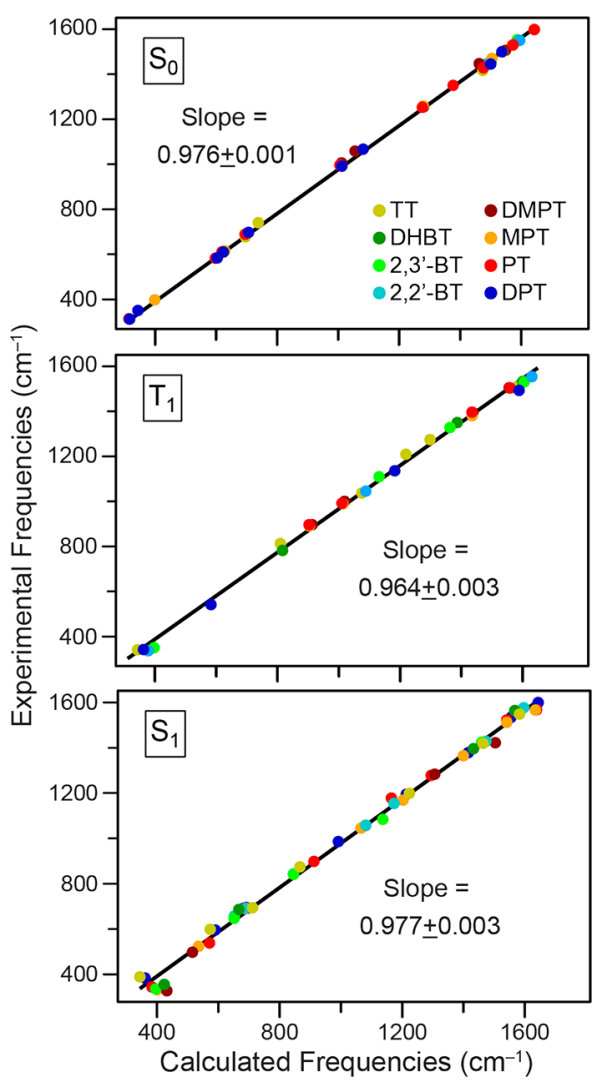

Figure 5. Calibration curves comparing the frequencies of the most prominent experimental bands with the calculated frequencies of the assigned transitions for $S_{0}, T_{1}$, and $S_{1}$. The slopes of the best-fit lines give the frequency scaling factors.

arguments from above. ${ }^{50}$ The transition energies of the three totally symmetric $\left(a_{1}\right)$ vibrations of DPT are indicated by vertical black bars immediately below the experimental and calculated spectra in Figure 6. The nuclear displacement vectors on the right side of the figure show that the calculated vibrations at $577 \mathrm{~cm}^{-1}\left(\nu_{18}\right)$ and $675 \mathrm{~cm}^{-1}\left(\nu_{25}\right)$ are combinations of in-phase phenyl ring distortions with the symmetric $\mathrm{C}-\mathrm{S}-\mathrm{C}$ bending and stretching modes of the thiophene ring, respectively. The phenyl motions are similar to the $6 a$ vibration in Wilson's notation for substituted benzene compounds, ${ }^{51}$ which typically has strong Raman activity. In contrast, the third $a_{1}$ vibration at $603 \mathrm{~cm}^{-1}$ involves a phenyl ring distortion that is similar to the $6 b$ vibration in Wilson's notation. ${ }^{51}$ The $6 b$ vibration typically has very weak Raman activity, consistent with our calculated spectrum for DPT.

In addition to the larger off-resonance Raman activities, modes $\nu_{18}$ and $\nu_{25}$ are more likely to be Franck-Condon active under $\pi$ excitation than the more localized motion of the 603 $\mathrm{cm}^{-1}$ mode $\left(\nu_{21}\right)$. We expect $\pi$ excitation to be delocalized across the entire molecule, therefore the delocalized modes are more likely to gain intensity in the resonance enhanced FSRS spectrum. Thus, we identify the experimental FSRS bands at 594 and $693 \mathrm{~cm}^{-1}$ as $\nu_{18}$ and $\nu_{25}$, respectively, on the basis of symmetry and resonance enhancement considerations. The different relative intensities of the transitions to $\nu_{18}$ and $\nu_{25}$ in the experimental and calculated $S_{1}$ spectra are probably a result of stronger resonance enhancement of the higher-frequency mode. A stronger enhancement of $\nu_{25}$ suggests that the upper state in the $S_{1}-S_{n}$ transition may have a larger displacement along the $\mathrm{C}-\mathrm{S}-\mathrm{C}$ stretching coordinate compared with $\mathrm{C}-\mathrm{S}-$ $\mathrm{C}$ bending. We tentatively assign the weak shoulder near 670 $\mathrm{cm}^{-1}$ in the experimental spectrum of DPT as an overtone or combination mode, on the basis of the intensity dependence as a function of excitation wavelength, described elsewhere. ${ }^{38}$

The band assignments are not as obvious for the lowersymmetry molecules PT and MPT, because fewer transitions can be excluded on the basis of symmetry alone. Nevertheless, we determine that the strongest experimental transitions in the $500-800 \mathrm{~cm}^{-1}$ region are not the same as the strongest transitions in the calculated off-resonant spectra for these two molecules. Specifically, we assign the dominant experimental bands of PT and MPT as the relatively weak calculated transitions at $679 \mathrm{~cm}^{-1}\left(\nu_{17}\right)$ and $670 \mathrm{~cm}^{-1}\left(\nu_{20}\right)$, respectively, on the basis of the strong correlation between experimental and calculated frequencies, as well as the similar nuclear motions compared with the $\nu_{25}$ mode of DPT.

The motions of the assigned vibrations for all three molecules, illustrated on the far right side of Figure 6, have similar phenyl deformation ( $6 a$ in Wilson's notation) and delocalized character that is likely to be Franck-Condon active. In contrast, the strongest transition in the calculated spectrum of $\operatorname{MPT}\left(\nu_{18}, 656 \mathrm{~cm}^{-1}\right)$ is primarily localized on the thiophene ring and includes significant displacement of the methyl group. Although the $656 \mathrm{~cm}^{-1}$ mode has more intensity in the offresonance spectrum, the $\pi$ conjugated electronic resonance is not likely to give a strong enhancement in this methyl rocking mode.

In the case of PT, the strongest calculated transition $\left(\nu_{14}\right)$ is more than $65 \mathrm{~cm}^{-1}$ below the experimental band, compared with only $11 \mathrm{~cm}^{-1}$ for the assigned mode, $\nu_{17}$. Furthermore, $\nu_{14}$ has an asymmetric distortion of the phenyl ring that is less likely to be Franck-Condon active than the symmetric $6 a$-type distortion of the assigned mode. The next closest totally symmetric $\left(a^{\prime}\right)$ vibration in the calculated spectrum is the mode at $586 \mathrm{~cm}^{-1}$, which has a $6 b$-type phenyl distortion (not represented in the figure) that is similar to the very weak 603 $\mathrm{cm}^{-1}$ mode in DPT. Neither of the $6 b$-type modes contribute to the FSRS spectra.

The other band with notable intensity in this region of the spectrum for PT is the calculated transition to $\nu_{11}$ at $558 \mathrm{~cm}^{-1}$. This mode is a combination of $\mathrm{C}-\mathrm{S}-\mathrm{C}$ bend and phenyl ring deformation that is similar to the $577 \mathrm{~cm}^{-1}\left(\nu_{18}\right)$ mode in DPT and $524 \mathrm{~cm}^{-1}\left(\nu_{13}\right)$ mode in MPT. All three of these calculated modes align well with weak transitions in the experimental spectra. Although the experimental bands associated with these assignments are consistently weaker than the higher frequency modes assigned above, these additional features support our overall assignment of the vibrations in the FSRS spectrum. The similar relative intensities of the two experimental bands for all 


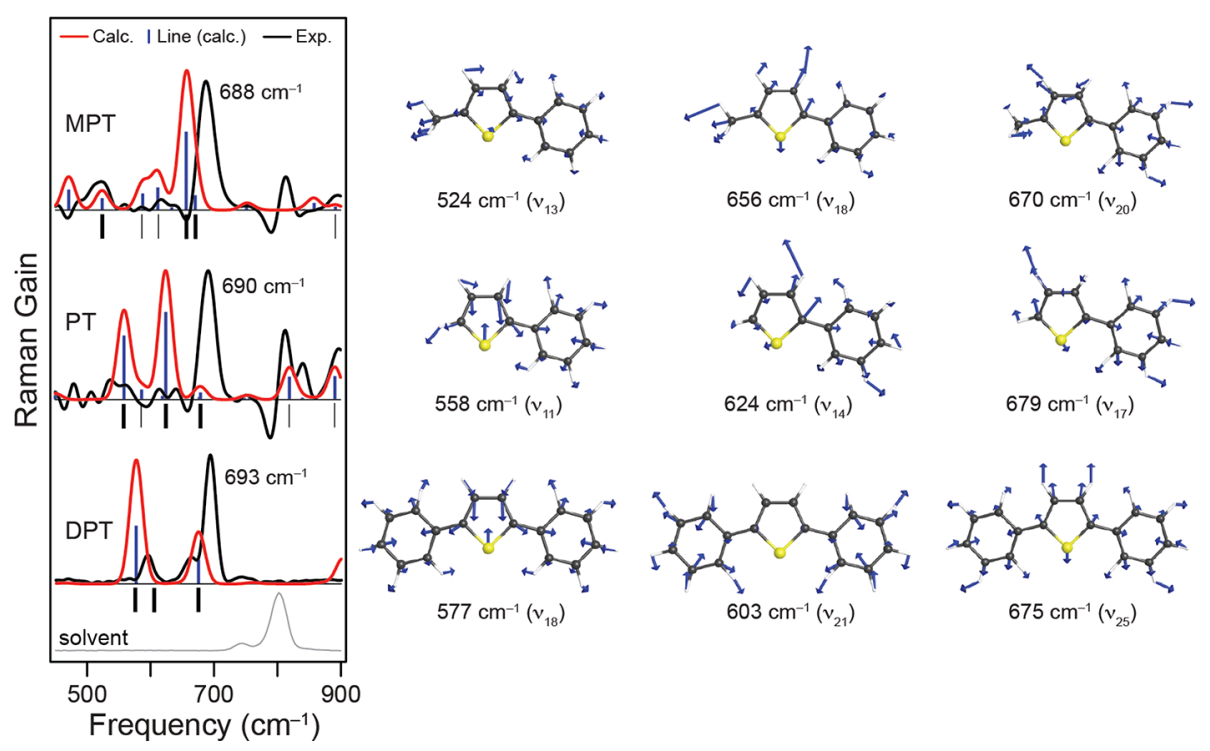

Figure 6. Comparison of the experimental (black) and calculated (red) $S_{1}$ spectra of MPT, PT, and DPT. Black bars under the spectra indicate which of the calculated modes are totally symmetric ( $a^{\prime}$ in PT and MPT; $a_{1}$ in DPT). The images on the right-hand side show vibrational displacement vectors for selected transitions that are indicated by bold black bars below the spectra.

three molecules show that resonance enhancement effects are conserved for vibrations with the same types of motion.

Finally, we note that the assignment of the $690 \mathrm{~cm}^{-1}$ experimental band in PT as $\nu_{17}$ rather than $\nu_{14}$ indicates that the $\pi$ resonance condition favors the localized $\mathrm{C}-\mathrm{S}$ stretching motion involving the unsubstituted carbon $\left(\mathrm{C}_{5}\right)$ rather than the phenyl-substituted carbon $\left(\mathrm{C}_{2}\right)$. Interestingly, the aryl-substituted $\mathrm{C}-\mathrm{S}$ stretching motion has been implicated in the rapid ISC of phenyl- and thienyl-substituted thiophenes. ${ }^{30,}$ This subtle distinction between modes $\nu_{14}$ and $\nu_{17}$ of PT highlights the importance of accurately assigning transitions in experimental FSRS spectra. Mode $\nu_{14}$ should be a better probe of the PT deactivation coordinate $\left(\mathrm{C}_{2}-\mathrm{S}\right.$ stretching $)$ compared with $\nu_{17}$. In fact, while a shift of the former vibration to lower frequency would indicate weakening of the $\mathrm{C}_{2}-\mathrm{S}$ bond, a concomitant shift to higher frequency would be expected for $\nu_{17}$ due to strengthening of the $\mathrm{C}_{5}-\mathrm{S}$ bond.

Our calculations here consider only the minimum energy geometry of the $S_{1}$ state of each molecule, however transient FSRS calculations have the potential to use these spectroscopic signatures to distinguish motions along the reaction coordinate. ${ }^{18}$ In fact, our assignments for MPT are consistent with the recently reported dynamics of that molecule, where the intense band near $690 \mathrm{~cm}^{-1}$ was observed to shift slightly to higher energy following the initial excitation. Notably, the shift to higher energy is consistent with the expected strengthening of the methyl-substituted $\mathrm{C}-\mathrm{S}$ bond, based on the prominent motion along that bond for mode $\nu_{20}$, rather than the weakening of the phenyl-substituted $\mathrm{C}-\mathrm{S}$ bond for $\nu_{18}$. The shift to higher energy therefore provides additional evidence that $\nu_{20}$ is the observed FSRS band, rather than $\nu_{18}$, even though the latter has the stronger calculated off-resonance intensity. Without considering resonance enhancements across all three compounds, it would be tempting to make incorrect assignments of the intense Raman bands of PT or MPT on the basis of the calculated intensities alone.

\section{CONCLUSIONS}

A key result of this work is that accurate excited-state resonance-enhanced Raman assignments are possible using relatively low-cost off-resonant calculations, provided the effects of the electronic resonance condition are taken into consideration. Importantly, the assignments are made possible by comparing the spectra for several molecules with similar structure. Transitions in the $500-800 \mathrm{~cm}^{-1}$ region of the $S_{1}$ spectra of DPT, PT, and MPT provide a representative example for making assignments on the basis of symmetry and resonance enhancement considerations across multiple compounds.

Most FSRS measurements rely on an electronic resonance condition to obtain increased Raman scattering signals, but there have not been any systematic studies examining how the resonance condition affects the assignment of experimental spectra before now. Our benchmark study shows that offresonance calculations can be deceiving, and highlights the importance of mode-specific resonance enhancement effects. While we have compared the experimental and theoretical Raman spectra for a series of aryl-substituted thiophene molecules, we expect similar results for other sets of structurally related compounds having similar excited-state absorption spectra.

We are currently working on a procedure to simulate excitedstate resonance Raman enhancements directly using the gradient approximation. ${ }^{38,52}$ After identifying the resonant $S_{n}$ states responsible for the excited-state absorption, this approach would obtain the mode-specific resonance enhancements by calculating the gradients of the relevant excited-state potential energy curves along the $S_{1}$ normal-mode coordinates. A comprehensive understanding of resonance enhancement effects will provide more detailed insight on the structural evolution of molecules in excited electronic states by allowing more precise tracking of the nuclear motions using FSRS spectra. 


\section{ASSOCIATED CONTENT}

\section{S Supporting Information}

The Supporting Information is available free of charge on the ACS Publications website at DOI: 10.1021/acs.jpca.7b09467.

Description of the method we use to deterime the optimum step size for numerical differentiation of the polarizability tensor; a description of the experimental baseline subtraction method for the experimental FSRS spectra; tables of calculated vibrational frequencies and Raman activities for the $S_{1}, T_{1}$, and $S_{0}$ states of each molecule; and tables with the optimized geometries for all three electronic states of each molecule (PDF)

\section{AUTHOR INFORMATION}

\section{Corresponding Authors}

*E-mail: mcaricato@ku.edu.

*E-mail: elles@ku.edu.

\section{ORCID}

David B. Williams-Young: 0000-0003-2735-3706

Marco Caricato: 0000-0001-7830-0562

\section{Notes}

The authors declare no competing financial interest.

\section{ACKNOWLEDGMENTS}

This research was supported by the American Chemical Society Petroleum Research Fund (53045-DNI6) and a National Science Foundation CAREER Award (CHE-1151555), with additional support from the General Research Fund and startup funding from the University of Kansas. CGE is grateful to Prof. Steven Corcelli (Notre Dame) for helpful discussions and providing preliminary excited-state calculations at an early stage of this work.

\section{REFERENCES}

(1) Kukura, P.; McCamant, D. W.; Mathies, R. A. Femtosecond Stimulated Raman Spectroscopy. Annu. Rev. Phys. Chem. 2007, 58, 461-488.

(2) Dietze, D. R.; Mathies, R. A. Femtosecond Stimulated Raman Spectroscopy. ChemPhysChem 2016, 17, 1224-1251.

(3) Kukura, P.; McCamant, D. W.; Davis, P. H.; Mathies, R. A. Vibrational Structure of the $S_{2}\left(1 \mathrm{~B}_{u}\right)$ Excited State of Diphenyloctatetraene Observed by Femtosecond Stimulated Raman Spectroscopy. Chem. Phys. Lett. 2003, 382, 81-86.

(4) Pontecorvo, E.; Ferrante, C.; Elles, C. G.; Scopigno, T. Structural Rearrangement Accompanying the Ultrafast Electrocyclization Reaction of a Photochromic Molecular Switch. J. Phys. Chem. B 2014, 118, 6915-6921.

(5) Lee, S. Y.; Heller, E. J. Time-Dependent Theory of Raman Scattering. J. Chem. Phys. 1979, 71, 4777-4788.

(6) Shin, K. K.; Zink, J. I. Quantitative Evaluation of the Relationships between Excited-State Geometry and the Intensities of Fundamentals, Overtones, and Combination Bands in Resonance Raman Spectra. Inorg. Chem. 1989, 28, 4358-4366.

(7) Kelley, A. M. Resonance Raman and Resonance Hyper-Raman Intensities: Structure and Dynamics of Molecular Excited States in Solution. J. Phys. Chem. A 2008, 112, 11975-11991.

(8) Lee, S. Y.; Zhang, D.; McCamant, D. W.; Kukura, P.; Mathies, R. A. Theory of Femtosecond Stimulated Raman Spectroscopy. J. Chem. Phys. 2004, 121, 3632-3642.

(9) Sun, Z.; Jin, Z.; Lu, J.; Zhang, D. H.; Lee, S.-Y. Wave Packet Theory of Dynamic Stimulated Raman Spectra in Femtosecond Pump-Probe Spectroscopy. J. Chem. Phys. 2007, 126, 174104.
(10) Wu, Y. C.; Zhao, B.; Lee, S. Y. Time-Dependent Wave Packet Averaged Vibrational Frequencies from Femtosecond Stimulated Raman Spectra. J. Chem. Phys. 2016, 144, 054104.

(11) Sun, Z.; Qiu, X. Q.; Lu, J.; Zhang, D. H.; Lee, S. Y. Three-State Model for Femtosecond Broadband Stimulated Raman Scattering. J. Raman Spectrosc. 2008, 39, 1568-1577.

(12) Dorfman, K. E.; Fingerhut, B. P.; Mukamel, S. Broadband infrared and Raman probes of excited-state vibrational molecular dynamics: simulation protocols based on loop diagrams. Phys. Chem. Chem. Phys. 2013, 15, 12348-12359.

(13) Dorfman, K. E.; Fingerhut, B. P.; Mukamel, S. Time-Resolved Broadband Raman Spectroscopies: A Unified Six-Wave-Mixing Representation. J. Chem. Phys. 2013, 139, 124113.

(14) Agarwalla, B. K.; Ando, H.; Dorfman, K. E.; Mukamel, S. Stochastic Liouville Equations for Femtosecond Stimulated Raman Spectroscopy. J. Chem. Phys. 2015, 142, 024115.

(15) Rao, B. J.; Gelin, M. F.; Domcke, W. Resonant Femtosecond Stimulated Raman Spectra: Theory and Simulations. J. Phys. Chem. A 2016, 120, 3286-3295.

(16) Liu, J.; Liang, W. Analytical Approach for the Excited-State Hessian in Time-Dependent Density Functional Theory: Formalism, Implementation, and Performance. J. Chem. Phys. 2011, 135, 184111.

(17) Petrone, A.; Lingerfelt, D.; Williams-Young, D.; Li, X. Ab Initio Transient Vibrational Spectral Analysis. J. Phys. Chem. Lett. 2016, 7, $4501-4508$

(18) Petrone, A.; Williams-Young, D. B.; Lingerfelt, D. B.; Li, X. Ab Initio Excited-State Transient Raman Analysis. J. Phys. Chem. A 2017, $121,3958-3965$.

(19) Egidi, F.; Williams-Young, D. B.; Baiardi, A.; Bloino, J.; Scalmani, G.; Frisch, M. J.; Li, X.; Barone, V. Effective Inclusion of Mechanical and Electrical Anharmonicity in Excited Electronic States: VPT2-TDDFT Route. J. Chem. Theory Comput. 2017, 13, 27892803.

(20) Neugebauer, J.; Reiher, M.; Kind, C.; Hess, B. Quantum Chemical Calculation of Vibrational Spectra of Large Molecules Raman and IR Spectra for Buckminsterfullerene. J. Comput. Chem. 2002, 23, 895-910.

(21) Sakamoto, A.; Tanaka, F.; Tasumi, M.; Torii, H.; Kawato, K.; Furuya, K. Comparison of the Raman Spectrum of trans-Stilbene in the $S_{1}$ State Calculated by the CIS Method and the Spectra Observed under Resonant and Off-Resonant Conditions. Vib. Spectrosc. 2006, 42, $176-182$.

(22) Tsumura, K.; Furuya, K.; Sakamoto, A.; Tasumi, M. Vibrational Analysis of trans-Stilbene in the Excited Singlet State by TimeDependent Density Functional Theory: Calculations of the Raman, Infrared, and Fluorescence Excitation Spectra. J. Raman Spectrosc. 2008, 39, 1584-1591.

(23) Dobryakov, A. L.; Ioffe, I.; Granovsky, A. A.; Ernsting, N. P.; Kovalenko, S. A. Femtosecond Raman Spectra of cis-Stilbene and trans-Stilbene with Isotopomers in Solution. J. Chem. Phys. 2012, 137, $244505-16$

(24) Grebner, D.; Helbig, M.; Rentsch, S. Size-Dependent Properties of Oligothiophenes by Picosecond Time-Resolved Spectroscopy. J. Phys. Chem. 1995, 99, 16991-16998.

(25) Lap, D.; Grebner, D.; Rentsch, S. Femtosecond time-resolved spectroscopic studies on thiophene oligomers. J. Phys. Chem. A 1997, $101,107-112$.

(26) Zheldakov, I. L.; Wasylenko, J. M.; Elles, C. G. Excited-State Dynamics and Efficient Triplet Formation in Phenylthiophene Compounds. Phys. Chem. Chem. Phys. 2012, 14, 6211-6218.

(27) Zhou, J.; Yu, W.; Bragg, A. E. Structural Relaxation of Photoexcited Quaterthiophenes Probed with Vibrational Specificity. J. Phys. Chem. Lett. 2015, 6, 3496-3502.

(28) Yu, W.; Magnanelli, T. J.; Zhou, J.; Bragg, A. E. Structural Heterogeneity in the Localized Excited States of Poly(3-hexylthiophene). J. Phys. Chem. B 2016, 120, 5093-5102.

(29) Bragg, A. E.; Yu, W.; Zhou, J.; Magnanelli, T. Ultrafast Raman Spectroscopy as a Probe of Local Structure and Dynamics in 
Photoexcited Conjugated Materials. J. Phys. Chem. Lett. 2016, 7, 3990-4000.

(30) Batignani, G.; Pontecorvo, E.; Ferrante, C.; Aschi, M.; Elles, C. G.; Scopigno, T. Visualizing Excited-State Dynamics of a Diaryl Thiophene: Femtosecond Stimulated Raman Scattering as a Probe of Conjugated Molecules. J. Phys. Chem. Lett. 2016, 7, 2981-2988.

(31) Yu, W.; Donohoo-Vallett, P. J.; Zhou, J.; Bragg, A. E. Ultrafast Photo-Induced Nuclear Relaxation of a Conformationally Disordered Conjugated Polymer Probed with Transient Absorption and Femtosecond Stimulated Raman Spectroscopies. J. Chem. Phys. 2014, 141, 044201.

(32) Wang, C.; Angelella, M.; Doyle, S. J.; Lytwak, L. A.; Rossky, P. J.; Holliday, B. J.; Tauber, M. J. Resonance Raman Spectroscopy of the $\mathrm{T}_{1}$ Triplet Excited State of Oligothiophenes. J. Phys. Chem. Lett. 2015, 6, 3521-3527.

(33) Thomas, A. K.; Brown, H. A.; Datko, B. D.; Garcia-Galvez, J. A.; Grey, J. K. Interchain Charge-Transfer States Mediate Triplet Formation in Purified Conjugated Polymer Aggregates. J. Phys. Chem. C 2016, 120, 23230-23238.

(34) Kölle, P.; Schnappinger, T.; de Vivie-Riedle, R. Deactivation pathways of thiophene and oligothiophenes: internal conversion versus intersystem crossing. Phys. Chem. Chem. Phys. 2016, 18, 7903-7915.

(35) Pontecorvo, E.; Kapetanaki, S.; Badioli, M.; Brida, D.; Marangoni, M.; Cerullo, G.; Scopigno, T. Femtosecond Stimulated Raman Spectrometer in the 320-520nm range. Opt. Express 2011, 19, 1107-1112.

(36) Pontecorvo, E.; Ferrante, C.; Elles, C. G.; Scopigno, T. Spectrally Tailored Narrowband Pulses for Femtosecond Stimulated Raman Spectroscopy in the Range 330-750 nm. Opt. Express 2013, 21, 6866-6872.

(37) Challa, J. R.; Du, Y.; McCamant, D. W. Femtosecond Stimulated Raman Spectroscopy Using a Scanning Multichannel Technique. Appl. Spectrosc. 2012, 66, 227-232.

(38) Quincy, T. J.; Barclay, M. S.; Caricato, M.; Elles, C. G. Probing Higher-Lying Electronic States with Mode-Specific Excited-State Resonance Raman Spectroscopy, in preparation, 2017.

(39) Becke, A. D. Density-Functional Thermochemistry 0.3. The Role of Exact Exchange. J. Chem. Phys. 1993, 98, 5648-5652.

(40) Becke, A. D. A New Mixing of Hartree-Fock and Local DensityFunctional Theories. J. Chem. Phys. 1993, 98, 1372-1377.

(41) Lee, C. T.; Yang, W. T.; Parr, R. G. Development of the ColleSalvetti Correlation-Energy Formula into a Functional of the ElectronDensity. Phys. Rev. B: Condens. Matter Mater. Phys. 1988, 37, 785-789.

(42) Dunning, T. H., Jr Gaussian Basis Sets for use in Correlated Molecular Calculations. I. The Atoms Boron through Neon and Hydrogen. J. Chem. Phys. 1989, 90, 1007-1023.

(43) Staniszewska, M.; Kupfer, S.; Łabuda, M.; Guthmuller, J. Theoretical Assessment of Excited State Gradients and Resonance Raman Intensities for the Azobenzene Molecule. J. Chem. Theory Comput. 2017, 13, 1263-1274.

(44) Frisch, M. J.; Trucks, G. W.; Schlegel, H. B.; Scuseria, G. E.; Robb, M. A.; Cheeseman, J. R.; Scalmani, G.; Barone, V.; Petersson, G. A.; Nakatsuji, H. et al. Gaussian Development Version, revision i.09 ed.; Gaussian, Inc.: Wallingford, CT, 2016.

(45) Lanzani, G.; Nisoli, M.; De Silvestri, S.; Tubino, R. Femtosecond Vibrational and Torsional Energy Redistribution in Photoexcited Oligothiophenes. Chem. Phys. Lett. 1996, 251, 339-345.

(46) NIST Computational Chemistry Comparison and Benchmark Database. 2016; http://cccbdb.nist.gov/, NIST Standard Reference Database Number 101.

(47) Scott, A.; Radom, L. Harmonic vibrational frequencies: An evaluation of Hartree-Fock, Möller-Plesset, quadratic configuration interaction, density functional theory, and semiempirical scale factors. J. Phys. Chem. 1996, 100, 16502-16513.

(48) Gorski, A.; Gawinkowski, S.; Herbich, J.; Krauss, O.; Brutschy, B.; Thummel, R. P.; Waluk, J. 1H-Pyrrolo[3,2-h]quinoline: A Benchmark Molecule for Reliable Calculations of Vibrational Frequencies, IR Intensities, and Raman Activities. J. Phys. Chem. A 2012, 116, 11973-11986.
(49) Castro, C. M.; Delgado, M. C. R.; Hernandez, V.; Hotta, S.; Casado, J.; Navarrete, J. T. L. Efficiency of the $\pi$ Conjugation in a Novel Family of $\alpha, \alpha^{\prime}$-bisphenyl End-capped Oligothiophenes by Means of Raman Spectroscopy. J. Chem. Phys. 2002, 116, 10419.

(50) Long, D. A. The Raman Effect: A Unified Treatment of the Theory of Raman Scattering by Molecules; John Wiley and Sons Ltd.: Baffins Lane, Chichester, 2002.

(51) Gardner, A. M.; Wright, T. G. Consistent Assignment of the Vibrations of Monosubstituted Benzenes. J. Chem. Phys. 2011, 135, 114305 .

(52) Heller, E. J.; Sundberg, R.; Tannor, D. Simple Aspects of Raman Scattering. J. Phys. Chem. 1982, 86, 1822-1833. 